\title{
Sexual compulsivity and its relationship with condomless sex among unmarried female migrant workers in Shanghai, China: a cross-sectional study
}

Mengyun Luo ${ }^{1 \dagger}$, Liping Zhu ${ }^{2+}$, Yuanyuan Dong ${ }^{1}$, Zezhou Wang ${ }^{1}$, Qiuming Shen ${ }^{1}$, Dandan $\mathrm{Mo}^{1}$, Li Du², Zhiruo Zhang ${ }^{1 *}$ and Yong Cai ${ }^{1 *}$ (I)

\begin{abstract}
Background: Individuals with high sexual compulsivity are preoccupied with their sexual desire to such an extent that it interferes with their normal daily life and can inhibit self-control. Previous studies have found a close association between sexual compulsivity and condomless sex among different populations; however, no studies have investigated this among unmarried female migrant workers in China. This study aimed to validate the Sexual Compulsivity Scale (SCS) for appropriate use and examine the association between sexual compulsivity and condomless sex in this target population.
\end{abstract}

Methods: In 2015, we recruited 1325 unmarried female migrant workers in Shanghai, China. Information about sociodemographics, sexual compulsivity, and condomless sex were collected using a structured questionnaire. Exploratory factor analysis and reliability analysis were performed to validate the Chinese version of the SCS. Multiple logistic regression analyses were used to examine the association between sexual compulsivity and condomless sex.

Results: The prevalence of condomless sex in the previous 6 months was $66.8 \%$ among all participants. The SCS was proven internally consistent for the overall scale (Cronbach's $a=0.89$ ), and two factors, Social Disruptiveness (Cronbach's $a=0.87$ ) and Perceived Self-Control (Cronbach's $a=0.84$ ), were extracted. With mean total score of 18.25 (standard deviation $=4.94$ ) after adjusting for significant sociodemographic factors, the SCS total scores (adjusted odds ratio $[\mathrm{AOR}]=1.04,95 \%$ confidence interval $[\mathrm{CI}]=1.02-1.07$ ) and two subscale scores $(A O R=1.07,95 \% \mathrm{Cl}=1.02-1.13 ; \mathrm{AOR}=1.06,95 \% \mathrm{Cl}=1.02-1.10)$ were all related to inconsistent condom use with partners in the previous 6 months.

Conclusions: The Chinese version of the SCS was found to be well adaptable for use among unmarried Chinese female migrant workers and a potential predictor for condomless sex. In addition to safe sex interventions, counselling on managing sexually compulsivity should also be provided in this population.

Keywords: Unmarried female migrant workers, Sexual compulsivity, Condomless sex, China

\footnotetext{
* Correspondence: zhangzhiruo@sjtu.edu.cn; caiyong202028@hotmail.com

${ }^{\dagger}$ Mengyun Luo and Liping Zhu contributed equally to this work.

'School of Public Health, Shanghai Jiao Tong University, No.227, South

Chongqing Road, Shanghai 200025, People's Republic of China

Full list of author information is available at the end of the article
}

(c) The Author(s). 2018 Open Access This article is distributed under the terms of the Creative Commons Attribution 4.0 International License (http://creativecommons.org/licenses/by/4.0/), which permits unrestricted use, distribution, and reproduction in any medium, provided you give appropriate credit to the original author(s) and the source, provide a link to the Creative Commons license, and indicate if changes were made. The Creative Commons Public Domain Dedication waiver (http://creativecommons.org/publicdomain/zero/1.0/) applies to the data made available in this article, unless otherwise stated. 


\section{Background}

Since 1978, socioeconomic transformation in the wake of China's opening-up policy has accelerated rural-to-urban migration in the country. As of 2015, there were approximately 247 million internal migrants in China [1]. Nearly half of these migrants were female [2], mostly young, unmarried workers aged around 20 to 24 years old [3].

The immense scale and high mobility of these migrants have raised many public health concerns, including high prevalence of sexually transmitted infections (STIs). Official data for 2015 showed that there were 22,162 new cases of STIs (including HIV/AIDS, hepatitis B virus, gonorrhea, and syphilis) in Shanghai, with migrants accounted for $26.77 \%$ of all STI cases and $36.97 \%$ of HIV/AIDS cases [4]. There is increasing concern that this large-scale migration might be contributing to the diffusion of STIs by acting as a bridge between low-risk and high-risk individuals in the general population [5-7].

Migrant workers in cities confront marginalized living conditions and inequitable access to medical insurance and health services [8]. Compared with original residents, internal migrants are at an unfair disadvantage in receiving sexual and reproductive health services. This is determined not only by the nature of service provision but also by the overarching social and political environments. On the social side, the vulnerable migrant population is isolated from comprehensive family planning services, and their reproductive health needs are mostly neglected. In the political arena, current national policy mainly targets married couples whereas counseling and services for unmarried women are limited [9].

As a result, many female migrants have insufficient knowledge about how to protect themselves from unwanted pregnancy. The primary indicator of unwanted pregnancy is having sex without use of a condom (condomless sex). Condomless sex and failure to use contraceptive methods reportedly leads to over $70 \%$ of unwanted pregnancies in China [10]. About 13 million abortions are induced annually in China, with $49.7 \%$ of these among young unmarried females [9-12]. According to a survey conducted in Guangdong Province, nearly one-third of female migrants have undergone induced abortions, $30 \%$ of which were illegal abortions at home or in private practice. Illegal abortions can cause particularly severe health consequences, including uterine perforation, intrauterine adhesions, and even death $[13,14]$.

Several studies have broadly assessed levels of knowledge, attitudes, and behaviors related to consistent condom use, as well as related psychosocial factors such as anxiety, depression, sexual addiction, and loneliness [15-19]. Kalichman [20] noted a relationship between the occurrence of unsafe sexual behavior and a propensity for losing self-control, as in the case of sexual compulsivity. Different from people with sexual addiction and hypersexuality, those with high sexual compulsivity are more likely to experience sexual disinhibition and uncontrolled sexual impulses [21]. Sexual compulsivity has been identified as an inhibitor of safe sexual practices, and might result in profound detrimental health consequences, including transmission of HIV and other sexually transmitted diseases [22-24].

Sexual compulsivity is an intriguing, though unpopular, topic in China as it is seldom discussed among the general public, in what is characteristically a sexually conservative culture. There has only been one study focusing on sexually active Chinese males, in which sexual compulsivity was not found to be associated with condomless sex [25]. However, unmarried females, who are particularly vulnerable to high-risk behavior, may feel greater shame in discussing compulsive sex behavior in China's largely patriarchal society [26]. Considering the high rate of unwanted pregnancy and induced abortion as a result of condomless sex, there is an urgent need to validate appropriate measures of sexual compulsivity for use among unmarried Chinese female migrant workers, to investigate the impact of sexual compulsivity on condomless sex. We hypothesized that: 1) the Sexual Compulsivity Scale (SCS) is well suited for use among unmarried female migrant workers in China; 2) sexual compulsivity is associated with condomless sex.

\section{Methods \\ Participants}

From August to October 2015, we conducted a cross-sectional study among unmarried female migrant workers in three factories in two industrialized areas of Shanghai. All unmarried female workers with any experience of premarital sex behavior were eligible for recruitment. Assuming a prevalence of inconsistent condom use during the previous 6 months of $60 \%[27,28]$, an $\alpha$ of 0.05 , and a relative sampling error of $0.1 \mathrm{P}$, we calculated a required sample size of 900 in case of sampling error and a nonresponse rate of $10 \%$. Of all women who met the criteria, 1325 volunteered to participate. Measures were self-reported in a private room with one investigator and one participant present. After satisfactorily completing the survey, each participant was paid 50 RMB (approximately 7 USD) for her participation.

\section{Measures \\ Sociodemographic variables}

A semi-structured questionnaire was used in this study. Sociodemographic information was collected including age, education level, and monthly income. In addition, participants were asked whether they had smoked tobacco in the previous 30 days (yes or no) and/or had received services related to HIV/AIDS prevention in the previous 6 months (yes or no). 


\section{Sexual compulsivity variable}

The 10-item SCS, designed by Kalichman in 1994 [20], was included in the survey. A four-point Likert-type scale ranging from 1 (strongly disagree) to 4 (strongly agree) was used for rating responses. Total scores ranged from 10 to 40, with a higher score indicating a higher degree of sexual compulsivity. The Chinese scale items were derived from a previous study of the JC School of Public Health and Primary Care, Chinese University of Hong Kong [25].

\section{Condomless sex variable}

Participants were additionally asked about the frequency of condom use during vaginal intercourse with their partners. Five response choices were given: every time, often, sometimes, seldom, and never. In this study, responses were recoded as a binary variable, with using a condom every time coded as "consistent condom use" and any other responses as "inconsistent condom use"; such response recoding has been widely used in other studies $[25,29]$. In the present study, "engaged in condomless sex" was defined as "inconsistent condom use" and was used as the main outcome and the dependent variable.

\section{Statistical analysis}

Mean and standard deviation (SD) were used for descriptive analysis. Exploratory factor analysis was performed using the principal components method with varimax rotation. Cronbach's $\alpha$ was used to assess internal reliability. Item analysis was performed by computing the corrected item-to-total correlation coefficients for the overall scale and the subscales. The associations between SCS score and condomless sex were investigated using univariate and adjusted odds ratios (AOR) with their respective 95\% confidence intervals (95\%CIs). We entered the total score and the two subscale scores separately in three different models. In addition, multiple logistic regression models were fit, adjusting for significant sociodemographic variables related to condomless sex in the unadjusted model. Statistical significance was defined as $p<0.05$, and IBM SPSS for Windows, Version 22.0 (IBM Corp., Armonk, NY, USA) was used for all statistical analyses.

\section{Ethical approval}

The study proposal was reviewed and approved by the Chinese National Nature Science Fund Committee. The study was also approved by the Ethics Committee of the School of Public Health, Shanghai Jiao Tong University.

\section{Results}

\section{Sociodemographic and behavior-related characteristics}

The 1325 participants were distributed among three age groups, with $35.0 \%$ aged over 23 years old. With respect to education, $69.8 \%$ reported receiving formal education for over 9 years. About two-thirds of the sample reported monthly income above 3200 RMB, 7.4\% had smoked tobacco in the previous 30 days, and $14.9 \%$ had received services related to HIV/AIDS prevention in the previous 6 months. Furthermore, 66.8\% of respondents reported engaging in vaginal intercourse without a condom in the previous 6 months (see Table 1).

\section{Survey responses on the SCS}

Table 2 shows the frequency distributions of the survey items. Over $20 \%$ of participants thought their sexual appetite had gotten in the way of their relationships (Item 1). Meanwhile, $17.2 \%$ of female migrant workers found themselves sometimes failing to meet their commitments and responsibilities because of their sexual behaviors (Item 4), and $16.7 \%$ felt that their sexual thoughts and feelings were stronger than they were (Item 7).

\section{Psychometric properties of the SCS}

Using the factor analysis procedure, we extracted two factors that explained $33.3 \%$ and $29.5 \%$ of variance (KaiserMeyer-Olkin Measure of Sampling Adequacy $=0.91 ; \chi^{2}$ of Bartlett's test of sphericity $=5970, p<0.001)$. The factor

Table 1 Personal characteristics of the study participants ( $n=$ 1325)

\begin{tabular}{|c|c|c|}
\hline & Number & Percent \\
\hline \multicolumn{3}{|l|}{ Socio-demographic variables } \\
\hline \multicolumn{3}{|l|}{ Age group } \\
\hline$\leq 21$ & 429 & 32.4 \\
\hline $21-23$ & 432 & 32.6 \\
\hline$>23$ & 464 & 35.0 \\
\hline \multicolumn{3}{|l|}{ Number of years of formal education } \\
\hline Primary education ( $\leq 9$ years) & 400 & 30.2 \\
\hline Secondary education (10-12 years) & 841 & 63.5 \\
\hline Tertiary eduation (>12 years) & 84 & 6.3 \\
\hline \multicolumn{3}{|l|}{ Monthly income (Yuan) } \\
\hline $\operatorname{Low}(\leq 3200)$ & 443 & 33.4 \\
\hline Medium (3201-4800) & 828 & 62.5 \\
\hline High (>4800) & 54 & 4.1 \\
\hline \multicolumn{3}{|l|}{ Behavior-related variables } \\
\hline \multicolumn{3}{|l|}{ Smoked tobacco in the past 30 days } \\
\hline Yes & 98 & 7.4 \\
\hline No & 1227 & 92.6 \\
\hline \multicolumn{3}{|l|}{$\begin{array}{l}\text { Having received services related to AIDS } \\
\text { prevention in the previous } 6 \text { months }\end{array}$} \\
\hline Yes & 198 & 14.9 \\
\hline No & 1127 & 85.1 \\
\hline \multicolumn{3}{|c|}{ Engaged in condomless sex in the previous 6 months } \\
\hline Yes & 885 & 66.8 \\
\hline No & 440 & 33.2 \\
\hline
\end{tabular}


Table 2 Item responses on the Sexual Compulsivity Scale $(n=1325)$

\begin{tabular}{|c|c|c|c|c|c|c|}
\hline \multirow[t]{2}{*}{ Items } & \multicolumn{4}{|l|}{ N (\%) } & \multirow[t]{2}{*}{ M } & \multirow[t]{2}{*}{ SD } \\
\hline & Strongly agree & Agree & Disagree & Strongly disagree & & \\
\hline 1. My sexual appetite has gotten in the way of my relationships & 19(1.4) & 258(19.5) & $687(51.8)$ & $361(27.2)$ & 2.0 & 0.7 \\
\hline 2. My sexual thoughts and behaviors are causing problems in my life & $14(1.1)$ & $162(12.2)$ & $772(58.3)$ & $377(28.5)$ & 1.9 & 0.7 \\
\hline 3. My desires to have sex have disrupted my daily life & $20(1.5)$ & 151(11.4) & $652(49.2)$ & $502(37.9)$ & 1.8 & 0.7 \\
\hline $\begin{array}{l}\text { 4. I sometimes fail to meet my commitments and responsibilities } \\
\text { because of my sexual behaviors }\end{array}$ & $32(2.4)$ & 196(14.8) & 639(48.2) & $458(34.6)$ & 1.9 & 0.8 \\
\hline 5. I sometimes get so horny I could lose control & $31(2.3)$ & $141(10.6)$ & $674(50.9)$ & $479(36.2)$ & 1.8 & 0.7 \\
\hline 6. I find myself thinking about sex while at work & $29(2.2)$ & $115(8.7)$ & $646(48.8)$ & $535(40.4)$ & 1.7 & 0.7 \\
\hline 7. I feel that sexual thoughts and feelings are stronger than I am & $34(2.6)$ & 187(14.1) & $716(54.0)$ & $388(29.3)$ & 1.9 & 0.7 \\
\hline 8. I have to struggle to control my sexual thoughts and behaviors & $27(2.0)$ & 185(14.0) & $702(53.0)$ & $411(31.0)$ & 1.9 & 0.7 \\
\hline 9. I think about sex more than I would like to & $29(2.2)$ & $94(7.1)$ & $759(57.3)$ & $443(33.4)$ & 1.8 & 0.7 \\
\hline $\begin{array}{l}\text { 10. It has been difficult for me to find sex partners who desire having } \\
\text { sex as much as I want to }\end{array}$ & $20(1.5)$ & $99(7.5)$ & $748(56.5)$ & 458(34.6) & 1.8 & 0.7 \\
\hline
\end{tabular}

loadings are shown in Table 3. The two sexual compulsivity factors were designated Social Disruptiveness (Items 1-4) and Perceived Self-Control (Items 5-10).

Acceptable levels of internal consistency were demonstrated for the SCS. Cronbach's $\alpha$ was 0.89 for the overall scale, 0.87 for the Social Disruptiveness dimension, and 0.84 for the Perceived Self-Control dimension. The item-to-total, item-to-subscale 1, and item-to-subscale 2 corrected correlation coefficients ranged from 0.54 to $0.74,0.65$ to 0.79 , and 0.59 to 0.65 , respectively.

\section{Relationship between SCS and condomless sex}

Medium monthly income, having smoked tobacco in the previous 30 days, SCS total score, Social Disruptiveness score, and Perceived Self-Control score were associated with condomless sex in the previous 6 months $(p<0.01)$.
After adjusting for the first two significant sociodemographic variables, greater likelihood to engage in condomless sex in the previous 6 months was still related to sexual compulsivity (AOR $=1.04,95 \% \mathrm{CI}=1.02-1.07)$ as well as Social Disruptiveness $(\mathrm{AOR}=1.07,95 \% \mathrm{CI}=$ $1.02-1.13)$ and Perceived Self-Control $(\mathrm{AOR}=1.06,95 \%$ $\mathrm{CI}=1.02-1.10)$ (see Table 4).

\section{Discussion}

Findings from our study demonstrated that two-thirds of the unmarried female migrant workers surveyed had engaged in vaginal intercourse without a condom in the previous 6 months, which was nearly in accordance with the results of other studies; our results were slightly higher than those for university students and unmarried youth but lower than the results for female sex workers

Table 3 Factor loadings, item-subscale, and item-total correlations of the Sexual Compulsivity Scale $(n=1325)$

\begin{tabular}{|c|c|c|c|c|}
\hline \multirow[t]{2}{*}{ Items } & \multicolumn{2}{|c|}{ Factor loadings } & \multicolumn{2}{|c|}{ Corrected correlation coefficients } \\
\hline & Factor 1 & Factor 2 & Item-total & Item-subscale \\
\hline \multicolumn{5}{|l|}{ Factor 1 (Social Disruptiveness) } \\
\hline 1. My sexual appetite has gotten in the way of my relationships & 0.82 & 0.12 & 0.54 & 0.65 \\
\hline 2. My sexual thoughts and behaviors are causing problems in my life & 0.82 & 0.23 & 0.64 & 0.73 \\
\hline 3. My desires to have sex have disrupted my daily life & 0.81 & 0.36 & 0.74 & 0.79 \\
\hline $\begin{array}{l}\text { 4. I sometimes fail to meet my commitments and responsibilities } \\
\text { because of my sexual behaviors }\end{array}$ & 0.77 & 0.33 & 0.67 & 0.71 \\
\hline \multicolumn{5}{|l|}{ Factor 2 (Perceived Self-Control) } \\
\hline 5. I sometimes get so horny I could lose control & 0.34 & 0.63 & 0.61 & 0.59 \\
\hline 6. I find myself thinking about sex while at work & 0.25 & 0.69 & 0.60 & 0.62 \\
\hline 7. I feel that sexual thoughts and feelings are stronger than I am & 0.11 & 0.76 & 0.55 & 0.61 \\
\hline 8. I have to struggle to control my sexual thoughts and behaviors & 0.31 & 0.67 & 0.62 & 0.62 \\
\hline 9. I think about sex more than I would like to & 0.17 & 0.77 & 0.61 & 0.65 \\
\hline $\begin{array}{l}\text { 10. It has been difficult for me to find sex partners who desire having } \\
\text { sex as much as I want to }\end{array}$ & 0.23 & 0.73 & 0.61 & 0.64 \\
\hline
\end{tabular}


Table 4 Factors associated with condomless sex among unmarried female migrant workers in Shanghai, China

\begin{tabular}{|c|c|c|c|}
\hline & $\mathrm{N}($ row\%)/Mean \pm Std. & ORu $(95 \% \mathrm{Cl})$ & AOR $(95 \% \mathrm{Cl})$ \\
\hline \multicolumn{4}{|l|}{ Age group } \\
\hline$\leq 21$ & $287(66.9)$ & 1 & \\
\hline $21-23$ & $301(69.7)$ & $1.14(0.85-1.52)$ & \\
\hline$>23$ & $297(64.0)$ & $0.88(0.67-1.16)$ & \\
\hline \multicolumn{4}{|c|}{ Number of years of formal education } \\
\hline $\operatorname{Low}(\leq 9)$ & $269(67.3)$ & 1 & \\
\hline Medium (10-12) & $565(67.2)$ & $1.00(0.77-1.29)$ & \\
\hline High (>12) & $51(60.7)$ & $0.75(0.46-1.22)$ & \\
\hline \multicolumn{4}{|l|}{ Monthly income (Yuan) } \\
\hline Low $(\leq 3200)$ & $274(61.9)$ & 1 & \\
\hline Medium (3201-4800) & $572(69.1)$ & $1.38(1.08-1.76) * *$ & \\
\hline High (>4800) & $39(72.2)$ & $1.60(0.86-3.00)$ & \\
\hline \multicolumn{4}{|c|}{ Tobacco use in the past 30 days } \\
\hline No & $804(65.5)$ & 1 & \\
\hline Yes & $81(82.7)$ & $2.51(1.47-4.28)^{* *}$ & \\
\hline \multicolumn{4}{|c|}{ Having received services related to AIDS prevention in the past 6 months } \\
\hline No & $743(65.9)$ & 1 & \\
\hline Yes & $142(71.7)$ & $1.31(0.94-1.83)$ & \\
\hline SCS total & $18.25 \pm 4.94$ & $1.05(1.03-1.08)^{* *}$ & $1.04(1.02-1.07)^{* *}$ \\
\hline Sexual Disruptiveness & $7.43 \pm 2.40$ & $1.09(1.03-1.14) * *$ & $1.07(1.02-1.13) * *$ \\
\hline Perceived Self-Control & $10.83 \pm 3.13$ & $1.08(1.04-1.12) * *$ & $1.06(1.02-1.10) * *$ \\
\hline
\end{tabular}

Dependent variable: condomless sex

AORs were adjusted for monthly income level and tobacco use in the previous 30 days

Abbreviations: $A O R$ adjusted odds ratio, OR odds ratio, $\mathrm{Cl}$ confidence interval, SCS Sexual Compulsivity Scale

** $p<0.01$

$[27,28,30,31]$. Owing to the high prevalence of condomless sex and the serious health consequence that might result, there is a clear need to provide this vulnerable population with sexual and reproductive health education.

To our knowledge, ours was the first study specifically targeting sexual compulsivity among unmarried female migrant workers in China, who do not receive sufficient attention with regard to their sexual and reproductive health. We found that the SCS was a highly reliable and valid tool for assessing sexual compulsivity in this target population. Moreover, we found that higher scores for sexual compulsivity as measured by the SCS were related to inconsistent condom use.

The 10-item SCS measure of sexual compulsivity was first developed by Kalichman in 1994 [20]. In the following years, both Kalichman [21] and McBride [32] identified two subscales, which they called Social Disruptiveness (Items 1-4) and Personal Discomfort (Items 5-10). However, the items were grouped differently in studies by Ballester-Arnal [33] and Liao [25]. Ballester-Arnal loaded the 10 items into two factors called Interference of Sexual Behavior (Items 1-4 and 10) and Failure to Control Sexual
Impulses (Items 5-9). In the study by Liao in Hong Kong, their modified Chinese version removed Item 4 and consisted of two factors named Functional Consequences (Items 1-3 and 10) and Controllability (Items 5-9). We doubted that the difference might be age and sex dependent, as Ballester's study mostly included college students aged 18-20 years, and Liao's only included Chinese males. The present study showed the same results as those of Kalichman and McBride [21, 32]. Adapting the scale items to local Chinese culture, these two dimensions are better labeled Social Disruptiveness and Perceived Self-Control. The present study serves as a reminder that measurement of sexual compulsivity must be culturally adapted and population-based.

Regarding the association between sexual compulsivity and condomless sex, we found that unmarried female migrant workers with high sexual compulsivity were more likely to engage in condomless sex. This was unsurprising, given that the same result has been reported for other target populations, including gays, lesbians, bisexual men and women, HIV-positive populations, and young college students [32-34]. Several theories could explain this phenomenon. As per the presently used 
scale, a higher score can be seen as: 1) preoccupation with one's sexual desire and sexual fantasy to a level that interferes with work and normal life; 2) a loss of control over obsessive thoughts and actual sexual behaviors [35, 36]. Individuals with difficulty controlling their impulses may be more likely to engage in condomless sex without considering potential negative outcomes such as STIs or unwanted pregnancy [37]. According to the theory of planned behavior, perceived behavioral control is one of the most important predictors of an individual's intention and behavior [38]. Evidence has showed that less perceived behavioral control over condom use is correlated with practicing condomless sex [39-45]. Meanwhile, attachment theory has postulated another perspective in that a real person cannot live up to the idealistic, imagined reality that a sexually compulsive/addictive person seeks [46]. Such people therefore tend to use sex as a means of comfort and anxiety reduction, without a need for emotional intimacy, which might also lead to condomless sex [47].

Our study results should be interpreted while considering several limitations. First, we cannot draw causal conclusions owing to the cross-sectional design of the study. Second, the clustered sampling method might result in selection bias; however, this method is commonly used for recruiting in highly aggregated populations, such as that in the present study. Third, given that the sample population was limited to one city, we cannot assert that is representative of all unmarried female migrant workers in China. Fourth, this study was limited in its reliance on self-report data because of the sensitive topic; however, we tried our best to obtain unbiased answers. Fifth, some other interesting elements, such as the type of male sex partners involved, intimate partner violence, and other unwanted sex including with employers or prospective employers, were not measured in this study; these could be included in further study.

Despite these limitations, given the large sample size, this report provides interesting insight on sexual compulsivity and its relationship with condomless sex in this relatively unique population. Further longitudinal research is needed to build on the findings of this study.

\section{Conclusions}

The Chinese version of the Sexual Compulsivity Scale was found to be suitable for use among unmarried Chinese female migrant workers and was evidently a potential predictor for condomless sex. This suggested that substantially more attention should be paid to implementing effective behavioral interventions among highly sexually compulsive female migrant workers in large cities. In addition to education about safe sex, counseling interventions should also be provided, to teach these women how to defuse sexually compulsive behavior and integrate healthy sexual behavior into their daily life.

\section{Abbreviations \\ AOR: Adjusted odds ratios; Cl: Confidence interval; SCS: Sexual Compulsivity Scale; SD: Standard deviation; STIs: Sexually transmitted infections}

\section{Acknowledgments}

We acknowledge the contribution of the investigators and staffs from Shanghai Jiao Tong University School of Public Health as well as Shanghai Center for Women and Children's Health who participated in the study. We thank Analisa Avila, ELS, of Liwen Bianji, Edanz Group China

(www.liwenbianji.cn/ac), for editing the English text of a draft of this manuscript.

\section{Funding}

This work was supported by the grants from the National Natural Science Foundation of China (71273174), the National Natural Science Foundation of China (71673187) and the Shanghai Key Discipline Construction Project in Public Health (15GWZK1002).

\section{Availability of data and materials}

The datasets used and/or analyzed during the current study are available from the corresponding author on reasonable request.

\section{Authors' contributions}

$M L, L Z, Y D, Z Z$ and $Y C$ developed this idea and designed overall research plans and methods. ZW, QS, DM and LD carried out the survey and analyzed data. $M L$ and $L Z$ wrote the paper. YD, YC and $Z Z$ revised the article for important intellectual content and approved the final version for submission. All authors reviewed and approved the final manuscript to be published.

Ethics approval and consent to participate

The study protocol was approved by the Ethics Committee of the School of Public, Shanghai Jiao Tong University. Before enrollment, all participants were given written informed consent after being told of the objectives and procedure of the study, as well as potential risks and benefits of participating. All procedures in the present study were conducted in accordance with the relevant guidelines and regulations.

Consent for publication

Not applicable.

\section{Competing interests}

The authors declare that they have no competing interests.

\section{Publisher's Note}

Springer Nature remains neutral with regard to jurisdictional claims in published maps and institutional affiliations.

\section{Author details}

${ }^{1}$ School of Public Health, Shanghai Jiao Tong University, No.227, South Chongqing Road, Shanghai 200025, People's Republic of China. ${ }^{2}$ Shanghai Center for Women and Children's Health, Shanghai 200062, People's Republic of China.

Received: 10 April 2018 Accepted: 17 October 2018 Published online: 09 November 2018

\section{References}

1. National Bureau of Statistics of China. Statistical Communiqué of the People's Republic of China on the 2015 National Economic and Social Development. http://www.stats.gov.cn/english/PressRelease/201602/ t20160229_1324019.html. Accessed 12 Dec 2016.

2. Lin T. Annual report on gender equality and Women's development in China 2008-2012. 1st ed. China: Social Sciences Academic Press; 2013.

3. Shen J, Huang Y. The working and living space of the 'floating population' in China. Asia Pac Viewp. 2003;44:51-62. 
4. Shanghai Municipal Commission of Health and Family Planning. Shanghai statutory report of infectious diseases. http://www.wsjsw.gov.cn/yqxx/ 20180815/59727.html. Accessed 12 Dec 2016.

5. Qian Z, Vermund SH, Wang N. Risk of HIV/AIDS in China: subpopulations of special importance. Sex Transm Infect. 2005;81:442-7.

6. Hu Z, Liu H, Li X, Stanton B, Chen X. HIV-related sexual behaviour among migrants and non-migrants in a rural area of China: role of rural-to-urban migration. Public Health. 2006;120:339-45.

7. Parrado EA, Flippen C. Community attachment, neighborhood context, and sex worker use among Hispanic migrants in Durham, North Carolina, USA. Soc Sci Med. 2010;70:1059-69.

8. He X, Wong DFK. A comparison of female migrant workers' mental health in four cities in China. Int J Soc Psychiatry. 2013;59:114-22.

9. Qian X, Tang S, Garner P. Unintended pregnancy and induced abortion among unmarried women in China: a systematic review. BMC Health Serv Res. 2004:4(1).

10. Tong $C$, Wu Y, Zhu H. Monitoring induced abortion in Shanghai childbearing women. Shanghai Med. 2002;25:451-4.

11. World Health Organization. Sexual and reproductive health of adolescents and youths in China: a review of literature and projects from 1995-2002. Manila: WHO Regional Office for the Western Pacific; 2005.

12. Chandra MV, Camacho AV, Michaud PA. WHO guidelines on preventing early pregnancy and poor reproductive outcomes among adolescents in developing countries. J Adolesc Health. 2013:52:517-22.

13. Jianduan $Z, W u L$, Junxin $S$. Situation and influencing factors of induced abortion of unmarried floating young women in Guangzhou City. Chinese JFamily Plann. 2006;14:671.

14. Hesketh T, Jun YX, Lu L, Mei WH. Health status and access to health care of migrant workers in China. Public Health Rep. 2008;123:189-97.

15. Derbyshire KL, Grant JE. Compulsive sexual behavior: a review of the literature. J Behav Addict. 2015:4:37-43.

16. Hill $L M$, Maman S, Kilonzo MN, Kajula LJ. Anxiety and depression strongly associated with sexual risk behaviors among networks of young men in Dar es Salaam, Tanzania. AIDS Care. 2017;29:252-8

17. Jackson JM, Seth P. DiClemente RJ, Lin A. Association of depressive symptoms and substance use with risky sexual behavior and sexually transmitted infections among African American female adolescents seeking sexual health care. Am J Public Health. 2015;105:2137-42.

18. Peixoto ERM, Barros FCR, Guimarães MDC. Factors associated with unprotected sexual practice among men and women with mental illnesses in Brazil. Cad Saude Publica. 2014:30:1475-86.

19. Anatale K, Kelly S. Factors influencing adolescent girls' sexual behavior: a secondary analysis of the 2011 youth risk behavior survey. Issues Ment Health Nurs. 2015;36:217-21.

20. Kalichman SC, Johnson JR, Adair V, Rompa D, Multhauf K, Kelly JA. Sexual sensation seeking: scale development and predicting AIDS-risk behavior among homosexually active men. J Pers Assess. 1994;62:385-97.

21. Kalichman SC, Cain D. The relationship between indicators of sexual compulsivity and high risk sexual practices among men and women receiving services from a sexually transmitted infection clinic. J Sex Res. 2004:41:235-41.

22. Muench F, Parsons JT. Sexual compulsivity and HIV: identification and treatment. Focus. 2004;19:1-5.

23. Satinsky S, Fisher C, Stupiansky N, Dodge B, Alexander A, Herbenick D, et al. Sexual compulsivity among men in a decentralized MSM community of the Midwestern United States. AIDS Patient Care STDs. 2008;22:553-60.

24. Benotsch EG, Kalichman SC, Kelly JA. Sexual compulsivity and substance use in HIV-seropositive men who have sex with men: Prevalence and predictors of high-risk behaviors. Addict Behav. 1999;24:857-68.

25. Liao W, Lau JT, Tsui HY, Gu J, Wang Z. Relationship between sexual compulsivity and sexual risk behaviors among Chinese sexually active males. Arch Sex Behav. 2015:44:791-8.

26. Lieber E, Chin D, Li L, Rotheramborus MJ, Detels R, Wu Z, et al. Sociocultural contexts and communication about sex in China: informing HIV/STD prevention programs. AIDS Educ Prev. 2009;21:415

27. Zheng Z, Zhou Y, Zheng L, Yang Y, Zhao D, Lou C, et al. Sexual behaviour and contraceptive use among unmarried, young women migrant workers in five cities in China. Reprod Health Matters. 2001;9:118-27.

28. Hong Y, Li X, Fang X, Zhao R. Depressive symptoms and condom use with clients among female sex workers in China. Sex Health. 2007:4:99-104.

29. Jing G, Yu B, Lau JT, Hao Y, Yu C, Zhou R, et al. Social environmental factors and condom use among female injection drug users who are sex workers in China. AIDS Behav. 2014;18:181-91.
30. Ma Q, Ono-Kihara M, Cong L, Pan X, Xu G, Zamani S, et al. Behavioral and psychosocial predictors of condom use among university students in eastern China. AIDS Care. 2009;21:249-59.

31. Chen H, Zhang L, Han Y, Lin T, Song X, Chen G, et al. HIV/AIDS knowledge, contraceptive knowledge, and condom use among unmarried youth in China. AIDS Care. 2012;24:1550-8.

32. McBride KR, Reece M, Sanders SA. Using the sexual compulsivity scale to predict outcomes of sexual behavior in young adults. Sex Addict Compulsivity. 2008;15:97-115.

33. Ballester AR, Gomez MS, Llario MD, Salmeron SP. Sexual compulsivity scale: adaptation and validation in the spanish population. J Sex Marital Ther. 2013;39:526-40

34. Kelly BC, Bimbi DS, Nanin JE, Izienicki H, Parsons JT. Sexual compulsivity and sexual behaviors among gay and bisexual men and lesbian and bisexual women. J Sex Res. 2009:46:301-8.

35. Schnarrs PW, Rosenberger JG, Satinsky S, Brinegar E, Stowers J, Dodge B, et al. Sexual compulsivity, the internet, and sexual behaviors among men in a rural area of the United States. AIDS Patient Care STDs. 2010;24:563-9.

36. Reece M, Dodge BM, McBride K. Sexual compulsivity: issues and challenges. In: Richard D, McAnulty M, Michele B, editors. Sex and sexuality: sexual function and dysfunction; 2006. p. 213-31.

37. Benotsch EG, Kalichman SC, Pinkerton SD. Sexual compulsivity in HIVpositive men and women: prevalence, predictors, and consequences of high-risk behaviors. Sex AddictCompulsivity. 2001;8:83-99.

38. Ajzen I. The theory of planned behavior. Organ Behav Hum Decis Process. 1991;50:179-211.

39. Wulfert E, Safren SA, Brown I, Wan CK. Cognitive, Behavioral, and personality correlates of HIV-positive Persons' unsafe sexual behavior. J Appl Soc Psychol. 1999;29:223-44

40. Robins AG, Dew MA, Kingsley LA, Becker JT. Do homosexual and bisexual men who place others at potential risk for HIV have unique psychosocial profiles? AIDS Educ Prev. 1997;9:239-51.

41. Godin G, Savard J, Kok G, Fortin C, Boyer R. HIV seropositive gay men: understanding adoption of safe sexual practices. AIDS Educ Prev. 1996;8:529.

42. Vroome EM, Wit JB, Stroebe W, Sandfort TG, Griensven GJ. Sexual behavior and depression among HIV-positive gay men. AIDS Behav. 1998;2:137-49.

43. Albarracin D, Johnson BT, Fishbein M, Muellerleile PA. Theories of reasoned action and planned behavior as models of condom use: a meta-analysis. Psychol Bull. 2001;127:142.

44. Buhi ER, Goodson P. Predictors of adolescent sexual behavior and intention: a theory-guided systematic review. J Adolesc Health. 2007;40:4-21.

45. Crepaz N, Marks G. Towards an understanding of sexual risk behavior in people living with HIV: a review of social, psychological, and medical findings. AIDS. 2002:16:135-49.

46. Weinstein A, Katz L, Eberhardt H, Cohen K, Lejoyeux M. Sexual compulsion-relationship with sex, attachment and sexual orientation. J Behav Addict. 2015;4:22-6

47. Roller CG. Sexually compulsive/addictive behaviors in women: a women's healthcare issue. J Midwifery Womens Health. 2007;52:486-91.

Ready to submit your research? Choose BMC and benefit from:

- fast, convenient online submission

- thorough peer review by experienced researchers in your field

- rapid publication on acceptance

- support for research data, including large and complex data types

- gold Open Access which fosters wider collaboration and increased citations

- maximum visibility for your research: over $100 \mathrm{M}$ website views per year

At BMC, research is always in progress.

Learn more biomedcentral.com/submissions 